\title{
KEYNOTE-716: Phase III study of adjuvant pembrolizumab versus placebo in resected high-risk stage II melanoma
}

\author{
Jason J Luke*,1, Paolo A Ascierto², Matteo S Carlino3,4,5,6, Jeffrey E Gershenwald7, \\ Jean-Jacques Grob ${ }^{8}$, Axel Hauschild ${ }^{9}$, John M Kirkwood ${ }^{1}$, Georgina V Long 5,6,10,11, Peter \\ Mohr ${ }^{12}$, Caroline Robert ${ }^{13,14}$, Merrick Ross ${ }^{7}$, Richard A Scolyer 5,6,15 iD, Charles H Yoon ${ }^{16}$, \\ Andrew Poklepovic ${ }^{17}$, Piotr Rutkowski ${ }^{18}$, James R Anderson ${ }^{19}$, Sama Ahsan ${ }^{19}$, Nageatte \\ Ibrahim $^{19}$ \& Alexander M M Eggermont ${ }^{13,20}$ \\ ${ }^{1}$ Cancer Immunotherapeutics Center, UPMC Hillman Cancer Center, University of Pittsburgh, Pittsburgh, PA 15232, USA \\ ${ }^{2}$ Melanoma, Cancer Immunotherapy and Development Therapeutics Unit, Istituto Nazionale Tumori IRCCS "Fondazione G. \\ Pascale," Naples, Italy \\ ${ }^{3}$ Department of Medical Oncology, Crown Princess Mary Cancer Centre, Westmead Hospital, Westmead, NSW, Australia \\ ${ }^{4}$ Blacktown Cancer and Haematology Centre, Blacktown Hospital, Blacktown, NSW, Australia \\ ${ }^{5}$ Melanoma Institute Australia, Sydney, NSW, Australia \\ ${ }^{6}$ Sydney Medical School, The University of Sydney, Sydney, NSW, Australia \\ ${ }^{7}$ Department of Surgical Oncology, The University of Texas MD Anderson Cancer Center, Houston, TX 77030, USA \\ ${ }^{8}$ Department of Dermatology and Skin Cancers, Hôpital de la Timone, Aix-Marseille Université, Marseille, France \\ ${ }^{9}$ Department of Dermatological Oncology, University Hospital Schleswig-Holstein, Kiel, Germany \\ ${ }^{10}$ Department of Medical Oncology, Mater Hospital, North Sydney, NSW, Australia \\ ${ }^{11}$ Department of Medical Oncology, Royal North Shore Hospital, St Leonards, NSW, Australia \\ ${ }^{12}$ Department of Dermatology, Elbe Kliniken Buxtehude, Buxtehude, Germany \\ ${ }^{13}$ Dermatology Unit, Department of Oncology, Gustave Roussy Cancer Centre, Villejuif, France \\ ${ }^{14}$ Department of Medicine, Université Paris-Sud, Orsay, France \\ ${ }^{15}$ Department of Medicine, Royal Prince Alfred Hospital, Sydney, NSW, Australia \\ ${ }^{16}$ Department of Surgical Oncology, Dana-Farber Cancer Institute, Boston, MA 02215, USA \\ ${ }^{17}$ Department of Internal Medicine, VCU Massey Cancer Center, Richmond, VA 23298, USA \\ ${ }^{18}$ Department of Soft Tissue/Bone Sarcoma and Melanoma, Maria Sklodowska-Curie Institute - Oncology Center, Warsaw, Poland \\ ${ }^{19}$ Department of Clinical Oncology, Merck \& Co., Inc., Kenilworth, NJ 07033, USA \\ ${ }^{20}$ Department of Medical Oncology, Gustave Roussy Cancer Institute \& Université Paris-Saclay, Paris, France \\ *Author for correspondence: Tel.: +1 412647 2811; lukejj@upmc.edu
}

Patients with high-risk stage II melanoma are at significant risk for recurrence after surgical resection. Adjuvant treatment options to lower the risk for distant metastases are limited. Although adjuvant IFN$\alpha 2 b$ is associated with improved relapse-free survival in patients with high-risk melanoma, toxicity and limited overall survival benefits limit its use. Adjuvant treatment with the PD-1 inhibitor pembrolizumab significantly improved recurrence-free survival, compared with placebo, in patients with resected stage III melanoma in the Phase III KEYNOTE-054 trial; efficacy in patients with stage II disease has not been established. This article describes the design and rationale of KEYNOTE-716 (NCT03553836), a two-part, randomized, placebo-controlled, multicenter Phase III study of adjuvant pembrolizumab in patients with surgically resected high-risk stage II melanoma.

Clinical trial registry \& ID: ClinicalTrials.gov, NCT03553836

First draft submitted: 29 October 2019; Accepted for publication: 4 December 2019; Published online: 24 December 2019

Keywords: adjuvant therapy $\bullet$ high-risk stage II melanoma • immune checkpoint inhibitor $\bullet$ pembrolizumab

Melanoma ranks 21 st among the most common cancers worldwide, with approximately 288,000 new cases and 61,000 deaths in 2018, and ranks fifth in the USA, with approximately 96,000 new cases and 7000 related deaths expected in 2019 [1,2]. The global incidence of cutaneous melanoma has increased at a faster rate than has any other 
malignancy [3], doubling between 1982 and 2011 in the USA [4]; 112,000 new melanoma cases are projected to occur in 2030 [4].

Therapeutic options for melanoma depend on host and tumor features (including stage, location and genetic profile) and may include surgical resection, radiotherapy, immunotherapy, targeted therapy or chemotherapy [5,6]. Patients with localized stage II melanoma are typically treated by surgery [7]; however, high-risk patients (stage IIB and IIC) often experience disease recurrence after surgical resection [8]. Among patients with stage IIB and IIC melanoma, 19 and $11 \%$, respectively, experience local recurrence; 45 and 58\%, experience regional recurrence and 44 and 39\% have distant recurrence. Recurrence patterns for stage III disease indicate that although distant recurrence is more common than locoregional recurrence, many patients experience locoregional recurrence [9-11]. A meta-analysis involving 15 randomized trials of adjuvant IFN- $\alpha$ in patients with resected high-risk melanoma showed reduced risk for relapse (improvement in recurrence-free survival [RFS]; hazard ratio of 0.88) and improved overall survival (OS; $\sim 3 \%$ OS advantage at 5 years) [12]. Although adjuvant IFN- $\alpha$ is offered to patients with stage II melanoma in some European countries, it is often not recommended because of significant associated toxicity [13], and its use may be limited to patients with ulcerated primary melanomas [14]. Furthermore, it has been reported that patients with melanoma experience fear of recurrence after being treated for localized melanoma [15]. There remains a significant need for adjuvant therapies to improve survival in patients with high-risk clinically localized melanoma.

Recent clinical trials in the adjuvant setting in melanoma have focused on treating high-risk stage III patients with the PD-1 inhibitors pembrolizumab and nivolumab or with combination BRAF and MEK inhibitors to decrease the risk for recurrence and improve distant metastasis-free survival (DMFS) and OS (European Organisation for Research and Treatment of Cancer [EORTC] 1325/KEYNOTE-054, NCT02362594 [16]; S1404/KEYNOTE053, NCT02506153 [17]; CheckMate 238, NCT02388906 [18]; COMBI-AD, NCT01682083 [19]). So far, the tolerability and benefits of adjuvant PD-1 inhibitor therapy have been reported in two studies $[16,18]$. CheckMate 238 showed 1 -year RFS rates of $71 \%$ with adjuvant nivolumab versus $61 \%$ with adjuvant ipilimumab in patients with resected stage III or IV melanoma [18]. Results of the EORTC 1325/KEYNOTE-054 study demonstrated 1-year RFS rates of $75 \%$ with pembrolizumab versus $61 \%$ with placebo in patients with resected stage III melanoma [16]. Moreover, preliminary findings of both studies indicate that DMFS may also be improved with these agents, with events reported in $25.2 \%$ of patients receiving nivolumab versus $31.4 \%$ of those receiving ipilimumab and in $15.2 \%$ of those receiving pembrolizumab versus $27.3 \%$ of those receiving placebo $[16,18]$. In COMBI-AD, the combination of dabrafenib (BRAF inhibitor) plus trametinib (MEK inhibitor) as adjuvant therapy provided a 3-year RFS rate of 58 versus $39 \%$ for adjuvant placebo in patients with stage III, $B R A F^{\mathrm{V} 600 \mathrm{E} / \mathrm{V} 600 \mathrm{~K}}$-mutant melanoma, as well as a 3 -year OS rate of 86 versus $77 \%$ and a suggestion of improved DMFS, with events reported in $25 \%$ versus $35 \%$ with adjuvant placebo [19]. Importantly, health-related quality of life was maintained with adjuvant pembrolizumab versus placebo in the KEYNOTE-054 study [20]. These encouraging data warrant further investigation of adjuvant pembrolizumab in patients with high-risk stage II melanoma.

\section{Introduction to the KEYNOTE-716 trial}

Herein, we describe the design of the randomized, placebo-controlled, parallel-group, crossover/rechallenge, multicenter Phase III KEYNOTE-716 study (ClinicalTrials.gov, NCT03553836), which is being conducted to evaluate the clinical benefit of adjuvant pembrolizumab therapy compared with placebo in pediatric patients (aged 12 to $<18$ years) and adult patients (aged $\geq 18$ years) with high-risk stage II melanoma.

\section{Background \& rationale}

Pembrolizumab is a highly selective anti-PD- 1 humanized monoclonal antibody that blocks the interaction between PD-1 and its ligands, PD-L1 and PD-L2 [21], thus releasing PD-1 pathway-mediated inhibition of immune response, including the antitumor response. The robust and durable antitumor activity and manageable safety profile of pembrolizumab demonstrated in several advanced solid tumors have led to the approval of pembrolizumab in numerous countries for the treatment of one or more advanced cancers, including melanoma [21,22].

The approval of pembrolizumab for the treatment of unresectable or metastatic melanoma in pretreated and in treatment-naive patients was based on results from the KEYNOTE-001 (ClinicalTrials.gov identifier, NCT01295827), KEYNOTE-002 (ClinicalTrials.gov identifier, NCT01704287) and KEYNOTE-006 (ClinicalTrials.gov identifier, NCT01866319) trials [23-25]. More recently, pembrolizumab was approved for the adjuvant treatment of patients with melanoma with involvement of lymph nodes after complete resection, based 
on results from the EORTC 1325/KEYNOTE-054 study [16]. In that study, adult patients with high-risk stage III melanoma who received 18 doses ( $\sim 1$ year) of pembrolizumab $200 \mathrm{mg}$ every 3 weeks after resection had a significantly longer RFS than those who received placebo. The 1-year RFS rate was $75.4 \%$ (95\% CI: 71.3-78.9\%) versus $61.0 \%$ (95\% CI: $56.5-65.1 \%$ ), respectively; hazard ratio for recurrence or death was 0.57 (98.4\% CI: $0.43-0.74 ; \mathrm{p}<0.001$ ). Grade $\geq 3$ treatment-related adverse events (AEs) occurred in $14.7 \%$ of patients in the pembrolizumab arm versus $3.4 \%$ in the placebo arm. The safety profile of pembrolizumab was consistent with that established through previous studies [16]. Moreover, the EORTC 1325/KEYNOTE-054 study showed a consistent reduction in risk for recurrence with pembrolizumab across a variety of subgroups, including microscopic and macroscopic positive lymph nodes and stages (IIIA [patients with stage N1a melanoma were required to have at least one micrometastasis measuring $>1 \mathrm{~mm}$ in greatest diameter], IIIB and IIIC), according to the AJCC Cancer Staging Manual, 7th edition (AJCC-7) [11,16]. This consistency across subgroups is maintained when using the AJCC Cancer Staging Manual, 8th edition (AJCC-8) [26] compared with the AJCC-7 staging system [27]. The EORTC 1325/KEYNOTE-054 study is also investigating whether pembrolizumab rechallenge benefits stage III patients who experience documented disease recurrence $>6$ months after completion of 1 year of adjuvant pembrolizumab [16,28]. It has been demonstrated that, in some advanced melanoma patients who derived benefit from anti-PD-1 or anti-PD-L1 therapy, rechallenge with the same agent after subsequent progression can confer additional benefit [29].

Adjuvant pembrolizumab may reduce the risk for recurrence in patients with surgically resected high-risk stage II melanoma because of the proven clinical benefit of adjuvant pembrolizumab in patients with resected stage III melanoma [16]. Patients with stage IIB, IIC or stage IIIB melanoma have similar survival outcomes (5-year melanoma-specific survival rates are 87,82 and $83 \%$ for stage IIB, IIC and IIIB, respectively; 10-year melanomaspecific survival rates are 82,75 and $77 \%$ for stage IIB, IIC and IIIB, respectively [26] $[9,10]$. Therefore, the evidence supports the study of adjuvant pembrolizumab in these high-risk patients and provides the opportunity to assess rechallenge with pembrolizumab in the event of disease recurrence in this patient population.

Stage II melanoma affects adult and pediatric patients. Pembrolizumab was well tolerated in pediatric patients, as demonstrated in the KEYNOTE-051 study, which established the recommended Phase II dose of $2 \mathrm{mg} / \mathrm{kg}$ every 3 weeks for children with advanced solid tumors, including melanoma or lymphoma [30]. Therefore, pembrolizumab has the potential to decrease the risk for recurrence and improve DMFS and OS in pediatric patients with newly diagnosed and resected high-risk stage II melanoma.

\section{Study design}

KEYNOTE-716 is a two-part (adjuvant and rechallenge/crossover), randomized, placebo-controlled, parallelgroup, multicenter, Phase III study of adjuvant pembrolizumab in adult patients (aged $\geq 18$ years) and pediatric patients (aged 12 to $<18$ years) with resected stage IIB or IIC cutaneous melanoma (Figure 1). Stage IIB and IIC cutaneous melanoma are defined as T category T3b and T4a, and T4b, respectively (Table 1), with negative sentinel lymph node biopsy, no regional metastases and no evidence of distant metastasis (per AJCC-8 [26]).

In Part 1, eligible patients will be randomly assigned in a 1:1 ratio to receive adjuvant therapy with either the pembrolizumab adult (aged $\geq 18$ years) dose of $200 \mathrm{mg}$ iv. every 3 weeks or the pediatric (aged $\geq 12$ to $<18$ years) dose of $2 \mathrm{mg} / \mathrm{kg}$ iv. up to a maximum of $200 \mathrm{mg}$ every 3 weeks or saline placebo intravenously every 3 weeks. Treatment will continue for up to 17 cycles. Part 2 is the unblinded crossover/rechallenge phase of the study in which eligible patients with disease recurrence can receive further treatment with pembrolizumab if they meet eligibility criteria.

Treatment allocation/randomization will occur centrally using an interactive response technology system. The patients and the investigator involved in administering the study treatments or clinical evaluation of the patients will be blinded to the group assignments in Part 1.

\section{Eligibility criteria}

A full account of the eligibility criteria is provided in Table 1 . In brief, male and female patients aged $\geq 12$ years will be eligible for enrollment if they have surgically resected and histologically or pathologically confirmed stage IIB or IIC cutaneous melanoma. 


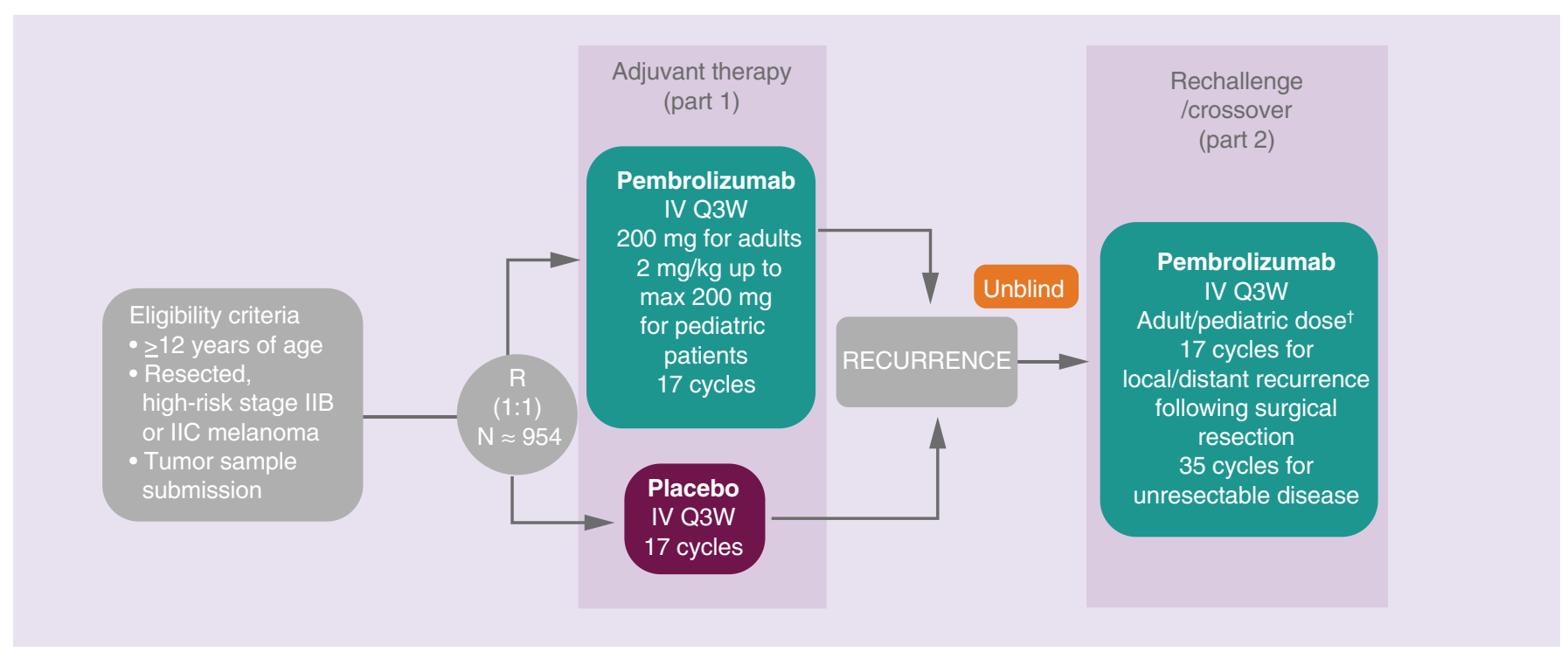

Figure 1. Study design.

${ }^{\dagger}$ Adult dose, $200 \mathrm{mg}$ Q3W; pediatric dose, $2 \mathrm{mg} / \mathrm{kg}$ Q3W (to a maximum of $200 \mathrm{mg} \mathrm{Q3W}$ ).

IV: Intravenous; Q3W: Every 3 weeks; R: Randomized.

\section{Planned sample size \& study period}

Approximately 954 eligible patients will be enrolled in the trial. The sample size is driven by the primary end point of RFS. The final analysis of RFS is event driven and will be conducted after approximately 179 RFS events have been observed, at about 48 months after enrollment starts, unless the study is terminated early. The trial start date was 12 September 2018, and the estimated study completion date is 21 October 2033.

\section{Study procedures}

Patients will undergo imaging that includes full chest/abdomen/pelvis computed tomography and/or MRI in Part 1, every 6 months while treatment is ongoing, at the end of treatment, every 6 months from years $2-4$ from randomization and then once in year 5 from randomization or until disease recurrence. For patients in Part 2, tumor imaging will be performed every 12 weeks during treatment for 1 or 2 years based on stage or recurrence pattern and resectability of disease before entry to Part 2. During Part 2 follow-up, the imaging schedule will be based on disease stage/recurrence pattern and resectability of disease before entry to Part 2. Response Evaluation Criteria in Solid Tumors, version 1.1 (RECIST 1.1 [31]), with a modification to allow a maximum of ten target lesions in total and five target lesions per organ will be used as the primary measure for assessment of tumor response and date of disease progression, and as a basis for all protocol guidelines related to disease status in Part 1. Objective response (partial response/complete response) will be confirmed based on repeat imaging using RECIST v1.1, and Guidelines for Response Criteria for Use in Trials Testing Immunotherapeutics (iRECIST) will be used to confirm progressive disease after initial site-assessed radiologic progressive disease per RECIST v1.1 in clinically stable patients. Patients who have disease recurrence will be unblinded. All patients who complete Part 1 pembrolizumab treatment will be eligible for additional cycles of pembrolizumab if they meet Part 2 rechallenge/crossover enrollment criteria (Table 1). Patients who received pembrolizumab during Part 1 and experience recurrence after more than 6 months from last dose of treatment may be eligible for rechallenge and receive up to 17 (after resection of recurrent disease, if feasible) or 35 cycles (for unresectable local or unresectable distant recurrence) of pembrolizumab in Part 2 (approximately 1 and 2 years of treatment, respectively). Patients are required to start Part 2 treatment within 4 weeks of recurrence. For safety, AEs will be monitored throughout the study and for 30 days after the end of treatment (90 days for serious AEs) and will be graded per Common Terminology Criteria for Adverse Events, version 4.0 [32].

In Part 1, the EuroQol 5 dimension, 5 level questionnaire (EQ-5D-5L) and EORTC Quality of Life Questionnaire Core 30 (QLQ-C30) will be completed electronically at baseline during treatment (Cycle 1), during treatment in year 1 (Cycles 5, 9, 13 and 17), every 12 weeks during year 2 and every 6 months during year 3. In 
Table 1. Eligibility criteria for Parts 1 and 2 of KEYNOTE-716.

\section{Part 1}

Key inclusion criteria

- Aged $\geq 12$ years

- Provision of informed consent by patient or legal representative

- Surgically resected and histologically/pathologically confirmed new diagnosis of stage IIB or IIC cutaneous melanoma ${ }^{\dagger}$

- Category T3b, T4a or T4b with pathologically confirmed negative sentinel lymph node biopsy specimen and no evidence of regional or distant metastatic disease ${ }^{\dagger}$

- Not previously treated for melanoma beyond complete surgical resection of current primary melanoma lesion

- No more than 12 weeks elapsed between full surgical resection and first dose of study treatment and with complete wound healing

- ECOG PS 0 or 1 (patients aged $>18$ years) or Lansky Play-Performance Scale score $\geq 50$ (children aged $\leq 16$ years) or Karnofsky Performance Status Scale score $\geq 50$ (children aged $>16$ and $<18$ years)

- Female patients of child-bearing potential and male patients must agree to follow the protocol's contraception guidance during the treatment period and for $\geq 120$ days thereafter

- Adequate hematologic function, defined as ANC $\geq 1500 / \mu \mathrm{l}$, platelet count $\geq 100,000 / \mu \mathrm{l}$ and hemoglobin $>9.0 \mathrm{~g} / \mathrm{dl}$ or $>5.6 \mathrm{mmol} / \mathrm{l}$

- Adequate renal function, defined as creatinine $\leq 1.5 \times$ ULN or measured or calculated creatinine clearance $>30 \mathrm{ml} / \mathrm{min}$ for those with creatinine levels $>1.5 \times$ ULN

- Adequate hepatic function, defined as total bilirubin $\leq 1.5 \times$ ULN or direct bilirubin $\leq$ ULN for those with total bilirubin levels $>1.5 \times$ ULN and ALT/AST levels $\leq 2.5 \times$ ULN (Parts 1 and $2:<5 \times$ ULN for patients in Part 2 with liver metastases)

- Adequate coagulation function, defined as INR $\leq 1.5 \times$ ULN unless the patient is receiving anticoagulant therapy as long as PT or aPTT is within the therapeutic range

Part 2 - Crossover or rechallenge after recurrence

Key inclusion criteria

- Investigator-determined/confirmed first disease recurrence $\mathbb{I}$ (radiologic or by examination/biopsy)

- Continuing from Part 1 placebo or completed 17 cycles of pembrolizumab treatment with no treatment delays in $>12$ weeks

- Full resection of lesions or biopsy of unresectable or metastatic disease confirmed by site pathologist to be melanoma ${ }^{\dagger \dagger}$

- ECOG PS $0-2$ in patients aged $\geq 18$ years or Lansky Play-Performance Scale score $\geq 50$ (children aged $\leq 16$ years) or Karnofsky Performance Status Scale score $\geq 50$ (children aged $>16$ and $<18$ years)

$\dagger$ According to AJCC Cancer Staging Manual, 8th edition [26].

${ }_{\ddagger}$ Basal carcinoma of the skin, squamous cell carcinoma of the skin, nonulcerated primary melanoma $<1 \mathrm{~mm}$ in depth with no nodal involvement or carcinoma in situ that has undergone potentially curative therapy is allowed.

$\S$ Replacement therapy (e.g., thyroxine, insulin, physiologic corticosteroid replacement therapy for adrenal or pituitary insufficiency) is not considered a form of systemic treatment and is allowed.

I Patients who are at $<6$ months from their last dose of pembrolizumab in Part 1 were not eligible.

\#Patients with focal or multifocal brain metastasis who are asymptomatic and do not require supraphysiologic steroid therapy are eligible and may receive concurrent radiation to these lesions.

${ }^{\dagger}$ If biopsy is clinically contraindicated, investigators should seek sponsor review and approval to start participant on Part 2 therapy. If participant has a thoracic lesion or lymph node suspected of recurrence (especially if solitary lesion), it is encouraged that a biopsy be performed to confirm metastatic melanoma vs another lung primary malignancy vs a nonmalignant lung disease (e.g., sarcoidosis).

AE: Adverse event; AJCC: American Joint Committee on Cancer; ANC: Absolute neutrophil count; aPTT: Activated partial thromboplastin time; ECOG PS: Eastern Cooperative Oncology Group performance status; GFR: Glomerular filtration rate; INR: International normalized ratio; PT: Prothrombin time; ULN: Upper limit of normal.

Part 2, the EQ-5D-5L will be completed at baseline (Cycle 1), during treatment (Cycles 9, 17 and 35) and at 24 and 48 weeks during the first year of treatment. Blood samples will be obtained to measure the pharmacokinetics of serum pembrolizumab in all pediatric patients. Biospecimens (i.e., blood components, tumor material and stool samples) for biomarker identification will be collected to support analyses of cellular components (e.g., protein, DNA, RNA and metabolites) and other circulating molecules.

\section{Outcome measures/end points}

The primary objective of the study is to compare RFS (assessed by the site investigator) between patients with completely resected high-risk stage II melanoma treated with adjuvant pembrolizumab and those receiving placebo. Secondary objectives are to compare DMFS (assessed by the site investigator) and OS between the two treatment arms and to assess the safety and tolerability of pembrolizumab. Exploratory objectives include comparison of change in global quality of life (QoL) using the QLQ-C30 global health status/QoL scale, characterization of 
health utilities using the EQ-5D-5L, comparison between treatment arms of the time to subsequent surgery or therapy and identification of molecular biomarkers that may predict response or resistance to treatment.

\section{Statistics}

Efficacy will be analyzed using the intention-to-treat population (all randomly assigned patients) by assigned treatment. The non-parametric Kaplan-Meier method will be used to estimate the RFS and OS in each treatment group. Nonparametric cumulative incidence curves will be used to estimate DMFS in each treatment arm. Treatment differences in RFS, DMFS and OS will be assessed by the stratified log-rank test. A stratified Cox proportional hazards model with the Efron method of tie handling will be used to assess the hazard ratio and its $95 \%$ CI for RFS, DMFS and OS.

Safety will be analyzed in all randomly assigned patients who received at least one dose of study medication according to the treatment received. Patient-reported outcomes will be analyzed in all patients for whom at least one patient-reported outcome assessment is available and who have received at least one dose of study treatment.

\section{Conclusion}

Because of the clinical benefit observed with adjuvant pembrolizumab in patients with stage III melanoma, a strong rationale exists to examine whether a similar benefit will be observed in adult and pediatric patients with high-risk resected stage II disease. Herein, we described the methodology of the KEYNOTE-716 study, a two-part (adjuvant and rechallenge/crossover), randomized, placebo-controlled, multicenter, Phase III study of adjuvant pembrolizumab in patients aged 12 years and older with resected stage IIB or IIC cutaneous melanoma. The results of this study will help to define the role of adjuvant pembrolizumab in the management of high-risk stage II melanoma, with potential to improve survival outcomes in this patient population.

\section{Executive summary}

- Treatment options that lower the risk for distant recurrence in patients with surgically resected high-risk stage II melanoma are limited.

- Adjuvant IFB- $\alpha 2 \mathrm{~b}$ has improved survival outcomes in a subset of patients with surgically resected high-risk stage II melanoma, but its use is limited by toxicity.

Background \& rationale

- Patients with localized stage I/II melanoma are typically treated by surgery.

- However, disease recurrence after surgery may occur, and there is an unmet need for adjuvant treatments options that can safely lower the risk for recurrence in high-risk subgroups of this patient population.

- Five-year melanoma-specific survival rates of patients with certain stage II and stage III melanoma subgroups are similar.

- Adjuvant pembrolizumab showed promising efficacy in patients with resected stage III melanoma in the KEYNOTE-054 study, in which recurrence-free survival was significantly longer with pembrolizumab than with placebo.

- Adjuvant pembrolizumab may reduce the risk for recurrence in patients with surgically resected high-risk stage II melanoma.

KEYNOTE-716 study design \& eligibility criteria

- KEYNOTE-716 is a two-part (adjuvant and rechallenge/crossover), randomized, placebo-controlled, multicenter, Phase III study of adjuvant pembrolizumab in patients 12 years of age and older with resected stage IIB or IIC cutaneous melanoma.

- In the double-blind phase (Part 1), patients will be randomly assigned 1:1 to receive pembrolizumab or placebo every 3 weeks for up to 17 cycles.

- In the unblinded phase (Part 2), patients with confirmed recurrence may be rechallenged (patients who received pembrolizumab in Part 1) or cross over to pembrolizumab (patients who received placebo in Part 1).

- Approximately 954 patients will be enrolled.

Outcome measures/end points

- The primary end point is recurrence-free survival; secondary end points are distant metastasis-free survival, overall survival and safety.

\section{Conclusion}

- This study will determine whether the benefits achieved with pembrolizumab adjuvant therapy seen in stage III and IV melanoma will also be observed in adult and pediatric patients with high-risk stage II melanoma. 
Supplementary data

To view the supplementary data that accompany this paper please visit the journal website at: www.futuremedicine.com/doi/suppl/10.2217/fon-2019-0666

\section{Acknowledgments}

The authors thank the patients and their families, investigators, co-investigators and the study teams at each of the participating centers.

Financial \& competing interests disclosure

Funding for this research was provided by Merck Sharp \& Dohme Corp., a subsidiary of Merck \& Co., Inc., Kenilworth, NJ, USA. JJ Luke serves on the Data and Safety Monitoring Board for TTC Oncology; reports advisory compensation from 7 Hills, Actym, Alphamab Oncology, Array, BeneVir, Mavu, Pyxis and Tempest; consulting compensation from AbbVie, Aduro, Astellas, AstraZeneca, Bayer, Bristol-Myers Squibb, Castle, CheckMate, Compugen, EMD Serono, IDEAYA, Immunocore, Incyte, Janssen, Jounce, Leap, Merck, Mersana, NewLink, Novartis, RefleXion, Spring Bank, Tempest and Vividion; research support (to institution) from AbbVie, Array (Scientific Research Agreement [SRA]), Boston Biomedical, BMS, Celldex, CheckMate (SRA), Compugen, Corvus, EMD Serono, Evelo (SRA), Delcath, Five Prime, FLX Bio, Genentech, Immunocore, Incyte, Leap, Medlmmune, Macrogenics, Novartis, Pharmacyclics, Palleon (SRA), Merck, Tesaro and Xencor; travel compensation from Array, AstraZeneca, Bayer, BeneVir, BMS, Castle, CheckMate, EMD Serono, IDEAYA, Immunocore, Janssen, Jounce, Merck, Mersana, NewLink, Novartis and RefleXion; and holds provisional patents serial \#15/612,657 (cancer immunotherapy), PCT/US18/36052 (microbiome biomarkers for antiPD-1/PD-L1 responsiveness: diagnostic, prognostic and therapeutic uses thereof). PA Ascierto reports research funds and fees for advisory boards for BMS, Roche-Genentech and Array Biopharma and fees for advisory boards for Merck Sharp \& Dohme, Amgen, Novartis, Merck Serono, Pierre Fabre, Incyte, Genmab, Newlink Genetics, Medlmmune, AstraZeneca, Syndax, Sun Pharma, Sanofi, Idera and Ultimovacs. MS Carlino reports advisory board compensation from MSD, BMS, Novartis and Pierre Fabre. JE Gershenwald reports advisory board compensation from Merck, Novartis, BMS and Syndax. J-J Grob reports advisory compensation from Roche, BMS, Novartis, MSD, Amgen, Pierre-Fabre, Sanofi, Pfizer and Merck. A Hauschild reports institutional research grants, speakers' honoraria and consultancy fees from Amgen, BMS, MSD, Merck, Pierre Fabre, Provectus, Regeneron, Roche, Sanofi-Genzyme and Novartis Pharma; institutional research grants and consultancy fees from Merck Serono and Philogen; and consultancy fees from OncoSec and Sun Pharma. JM Kirkwood received non-financial assistance with manuscript preparation from ArticulateScience LLC; institutional research grants from Prometheus; institutional research grants and personal fees from Merck and Immunocore; and personal fees from Array BioPharma, BMS, Novartis, Roche and Amgen. GV Long is consultant advisor to Aduro, Amgen, Array, BMS, MSD, Novartis, Pierre Fabre, Oncosec and Roche. P Mohr reports advisory board and speakers' bureau compensation from, BMS, GSK, MSD, Novartis, Pierre Fabre, Merck and Sanofi. C Robert reports advisory board compensation from BMS, MSD, Roche, Novartis, Pierre Fabre, Array, Sanofi, Merck, Amgen and Effector. M Ross reports honoraria and travel expenses from Amgen, Novartis, Castle Biosciences and Merck and consultancy compensation from Merck. RA Scolyer reports advisory board compensation from MSD, Novartis, Myriad and NeraCare. CH Yoon reports consultancy compensation from MSD. A Poklepovic reports advisory compensation from Merck and Castle Biosciences; consultancy compensation from Novartis; and speakers' bureau compensation from BMS. P Rutkowski has received honoraria for lectures and advisory board compensation from Novartis, MSD, BMS, Roche and Pierre Fabre and honoraria for lectures from Pfizer, Amgen and Eli Lilly. JR Anderson, S Ahsan and N Ibrahim report employment with Merck Sharp \& Dohme Corp., a subsidiary of Merck \& Co., Inc., Kenilworth, NJ, USA, and stock ownership in Merck \& Co., Inc., Kenilworth, NJ, USA. AMM Eggermont has received honoraria from Actelion, Biocad, Bioinvent, BMS, CatalYm, CellDex, Ellipses, Gilead, GSK, HalioDx, IO Biotech, ISA Pharmaceuticals, Medlmmune, MSD, Novartis, Polynoma, Regeneron, Sanofi, Sellas and SkylineDx; independent data monitoring committee compensation from Novartis and Pfizer; and holds equity in RiverD, SkylineDX and Theranovir. In addition, AMM Eggermont is the study chair of Adjuvant Pembrolizumab in Stage III melanoma Trial: EORTC 1325 / KEYNOTE-054, and Adjuvant Ipilimumab in stage III melanoma Trial: EORTC 18071. The authors have no other relevant affiliations or financial involvement with any organization or entity with a financial interest in or financial conflict with the subject matter or materials discussed in the manuscript apart from those disclosed.

Medical writing and/or editorial assistance was provided by J Kolston, PhD, of the ApotheCom pembrolizumab team (Yardley, PA, USA). This assistance was funded by Merck Sharp \& Dohme Corp., a subsidiary of Merck \& Co., Inc., NJ, USA.

\section{Ethical conduct of research}

The authors state that they have obtained appropriate institutional review board approval and have followed the principles outlined in the Declaration of Helsinki for all human experimental investigations. In addition, informed consent has been obtained from the patients involved. 


\section{Conference paper information}

This paper was previously presented at: 15th Annual Congress of the Society for Melanoma Research (SMR); 24-27 October 2018; Manchester, UK; 33rd Annual Meeting of the Society for Immunotherapy of Cancer (SITC 2018); 7-11 November 2018; Washington, DC, USA; ASCO-SITC Clinical Immuno-Oncology Symposium; 28 February-2 March 2019; San Francisco, CA, USA; 15th European Association of Dermato-Oncology (EADO) Congress; 24-27 April 2019; Paris, France; American Society of Clinical Oncology (ASCO) Annual Meeting; 1-5 June 2018; Chicago, IL, USA; 24th World Congress of Dermatology (WCD); 10-15 June 2019; Milan, Italy.

\section{Open access}

This work is licensed under the Attribution-NonCommercial-NoDerivatives 4.0 Unported License. To view a copy of this license, visit http://creativecommons.org/licenses/by-nc-nd/4.0/

\section{References}

Papers of special note have been highlighted as: $\bullet$ of interest; $\bullet \bullet$ of considerable interest

1. Bray F, Ferlay J, Soerjomataram I, Siegel RL, Torre LA, Jemal A. Global cancer statistics 2018: GLOBOCAN estimates of incidence and mortality worldwide for 36 cancers in 185 countries. CA Cancer J. Clin. 68(6), 394-424 (2018).

2. Siegel RL, Miller KD, Jemal A. Cancer statistics, 2019. CA Cancer J. Clin. 69(1), 7-34 (2019).

3. Ali Z, Yousaf N, Larkin J. Melanoma epidemiology, biology and prognosis. Eur. J. Cancer Suppl. 11(2), 81-91 (2013).

4. Guy GP Jr, Thomas CC, Thompson T et al. Vital signs: melanoma incidence and mortality trends and projections - United States, 1982-2030. MMWR Morb. Mortal. Wkly Rep. 64(21), 591-596 (2015).

5. Domingues B, Lopes JM, Soares P, Populo H. Melanoma treatment in review. ImmunoTargets Ther. 7, 35-49 (2018).

6. Luke JJ, Flaherty KT, Ribas A, Long GV. Targeted agents and immunotherapies: optimizing outcomes in melanoma. Nat. Rev. Clin. Oncol. 14(8), 463-482 (2017).

7. National Comprehensive Cancer Network. NCCN clinical practice guidelines in oncology - cutaneous melanoma (Version 2.2019). (2019). https://www.nccn.org/professionals/physician_gls/pdf/cutaneous_melanoma.pdf

8. Koster BD, Van Den Hout M, Sluijter BJR et al. Local adjuvant treatment with low-dose CpG-B offers durable protection against disease recurrence in clinical stage I-II melanoma: data from two randomized Phase II trials. Clin. Cancer Res. 23(19), 5679-5686 (2017).

9. Berger AC, Ollila DW, Christopher A et al. Patient symptoms are the most frequent indicators of recurrence in patients with American Joint Committee on Cancer stage II melanoma. J. Am. Coll. Surg. 224(4), 652-659 (2017).

10. Eggermont AMM, Chiarion-Sileni V, Grob JJ et al. Adjuvant ipilimumab versus placebo after complete resection of stage III melanoma: long-term follow-up results of the European Organisation for Research and Treatment of Cancer 18071 double-blind Phase III randomised trial. Eur. J. Cancer 119, 1-10 (2019).

11. Balch CM, Gershenwald JE, Soong SJ et al. Final version of 2009 AJCC melanoma staging and classification. J. Clin. Oncol. 27(36), 6199-6206 (2009).

12. Ives NJ, Suciu S, Eggermont AMM et al. Adjuvant interferon-alpha for the treatment of high-risk melanoma: an individual patient data meta-analysis. Eur. J. Cancer 82, 171-183 (2017).

- Meta-analysis of individual patient data from randomized trials of IFN- $\alpha$ in patients with high-risk melanoma demonstrated that adjuvant IFN- $\alpha$ significantly reduced the risk for relapse and improved survival with no additional benefit with higher versus lower doses.

13. Garbe C, Peris K, Hauschild A et al. Diagnosis and treatment of melanoma. European consensus-based interdisciplinary guideline Update 2016. Eur. J. Cancer 63, 201-217 (2016).

14. Eggermont AMM, Dummer R. The 2017 complete overhaul of adjuvant therapies for high-risk melanoma and its consequences for staging and management of melanoma patients. Eur. J. Cancer 86, 101-105 (2017).

15. Bell KJL, Mehta Y, Turner RM et al. Fear of new or recurrent melanoma after treatment for localised melanoma. Psychooncology 26(11), 1784-1791 (2017).

16. Eggermont AMM, Blank CU, Mandala M et al. Adjuvant pembrolizumab versus placebo in resected stage III melanoma. N. Engl. J. Med. 378(19), 1789-1801 (2018).

-• Randomized, double-blind Phase III trial (European Organisation for Research and Treatment of Cancer 1325) demonstrating that up to 1 year of adjuvant pembrolizumab ( $200 \mathrm{mg}$ every 3 weeks) conferred significantly longer recurrence-free survival with no new toxicity than placebo in patients with resected high-risk stage III melanoma.

17. Grossmann KF, Othus M, Tarhini AA et al. SWOG S1404: a Phase III randomized trial comparing high dose interferon to pembrolizumab in patients with high risk resected melanoma. J. Clin. Oncol. 33(Suppl.), TPS9085 (2015).

18. Weber J, Mandala M, Del Vecchio M et al. Adjuvant nivolumab versus ipilimumab in resected stage III or IV melanoma. N. Engl. J. Med. 377(19), 1824-1835 (2017). 
-• Randomized, double-blind Phase III trial (CheckMate 238) showed that adjuvant therapy with nivolumab significantly prolonged recurrence-free survival with reduced toxicity compared with adjuvant therapy with ipilimumab in patients undergoing resection of stage IIIB, IIIC or IV melanoma.

19. Long GV, Hauschild A, Santinami M et al. Adjuvant dabrafenib plus trametinib in stage III BRAF-mutated melanoma. N. Engl. J. Med. 377(19), 1813-1823 (2017).

•• Randomized, double-blind Phase III trial (COMBI-AD) found that adjuvant therapy with dabrafenib plus trametinib significantly reduced the risk for recurrence with no new toxicity versus adjuvant placebo in patients with stage III $B R A F$ V600E or $B R A F^{\mathrm{V} 600 \mathrm{~K}}$-mutant melanoma.

20. Coens C, Bottomley A, Blank CU et al. Health-related quality-of-life results for pembrolizumab versus placebo after complete resection of high-risk stage III melanoma from the EORTC 132. Ann. Oncol. 29(Suppl.), viii442-viii466 (2018).

21. KEYTRUDA ${ }^{\circledR}$ (pembrolizumab) for injection, for intravenous use. Merck Sharp \& Dohme Corp, NJ, USA (2019).

22. KEYTRUDA (pembrolizumab) $50 \mathrm{mg}$ powder for concentrate for solution for infusion (summary of product characteristics). Merck Sharp \& Dohme Limited, Hoddeston, UK (September 2019).

23. Ribas A, Hamid O, Daud A et al. Association of pembrolizumab with tumor response and survival among patients with advanced melanoma. JAMA 315(15), 1600-1609 (2016).

24. Ribas A, Puzanov I, Dummer R et al. Pembrolizumab versus investigator-choice chemotherapy for ipilimumab-refractory melanoma (KEYNOTE-002): a randomised, controlled, Phase II trial. Lancet Oncol. 16(8), 908-918 (2015).

- Randomized Phase II trial (KEYNOTE-002) that established pembrolizumab as a standard of care for the treatment of ipilimumab-refractory melanoma.

25. Robert C, Schachter J, Long GV et al. Pembrolizumab versus ipilimumab in advanced melanoma. N. Engl. J. Med. 372(26), 2521-2532 (2015).

26. Gershenwald JE, Scolyer RA, Hess KR et al. Melanoma staging: evidence-based changes in the American Joint Committee on Cancer eighth edition cancer staging manual. CA Cancer J. Clin. 67(6), 472-492 (2017).

27. Eggermont AMM, Blank CU, Mandala M et al. Prognostic and predictive value of AJCC-8 staging in the Phase III EORTC1325/KEYNOTE-054 trial of pembrolizumab vs placebo in resected high-risk stage III melanoma. Eur. J. Cancer 116, 148-157 (2019).

28. Adjuvant immunotherapy with anti-PD-1 monoclonal antibody pembrolizumab (MK-3475) versus placebo after complete resection of high-risk stage III melanoma: a randomized, double-blind Phase III trial of the EORTC Melanoma Group [protocol]. (2018). https://clinicaltrials.gov/ProvidedDocs/94/NCT02362594/Prot_SAP_000.pdf

29. Bernard-Tessier A, Baldini C, Martin P et al. Outcomes of long-term responders to anti-programmed death 1 and anti-programmed death ligand 1 when being rechallenged with the same anti-programmed death 1 and anti-programmed death ligand 1 at progression. Eur. J. Cancer 101, 160-164 (2018).

- Findings of this observational study suggest that patients who received benefit from anti programmed death ligand 1 therapy and progressed after completing or achieving a response to first-course anti programmed death ligand 1 therapy may benefit from rechallenge with the same immunotherapy.

30. Geoerger B, Kang HJ, Yalon-Oren M et al. KEYNOTE-051: an update on the Phase II results of pembrolizumab (pembro) in pediatric patients (pts) with advanced melanoma or a PD-L1-positive advanced, relapsed or refractory solid tumor or lymphoma. J. Clin. Oncol. 36(Suppl.), 10525 (2018).

31. Eisenhauer EA, Therasse P, Bogaerts J et al. New response evaluation criteria in solid tumours: revised RECIST guideline (version 1.1). Eur. J. Cancer 45(2), 228-247 (2009).

32. US Department of Health and Human Services. Common terminology ccriteria for adverse events (CTCAE) Version 4.0 (2009). https://evs.nci.nih.gov/ftp1/CTCAE/CTCAE_4.03/Archive/CTCAE_4.0_2009-05-29_QuickReference_8.5x11.pdf 


\section{Article details}

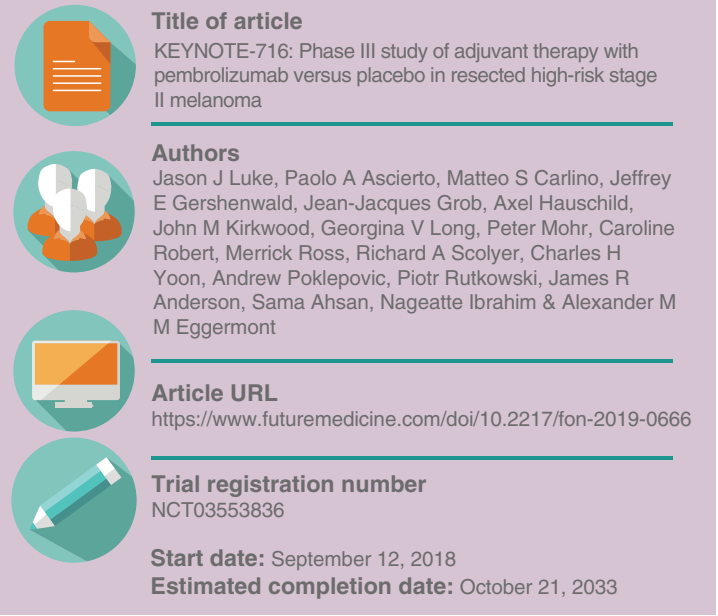

Primary objectives/rationale

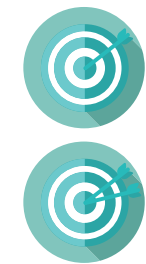

Primary objective

Compare between treatment arms RFS as assessed by the investigator

\section{Secondary key objectives}

Compare between treatment arms:

-DMFS as assessed by the investigator

- OS

- Safety and tolerability with respect to proportion of AEs

Study design and treatment including planned sample size, planned study period and study procedures

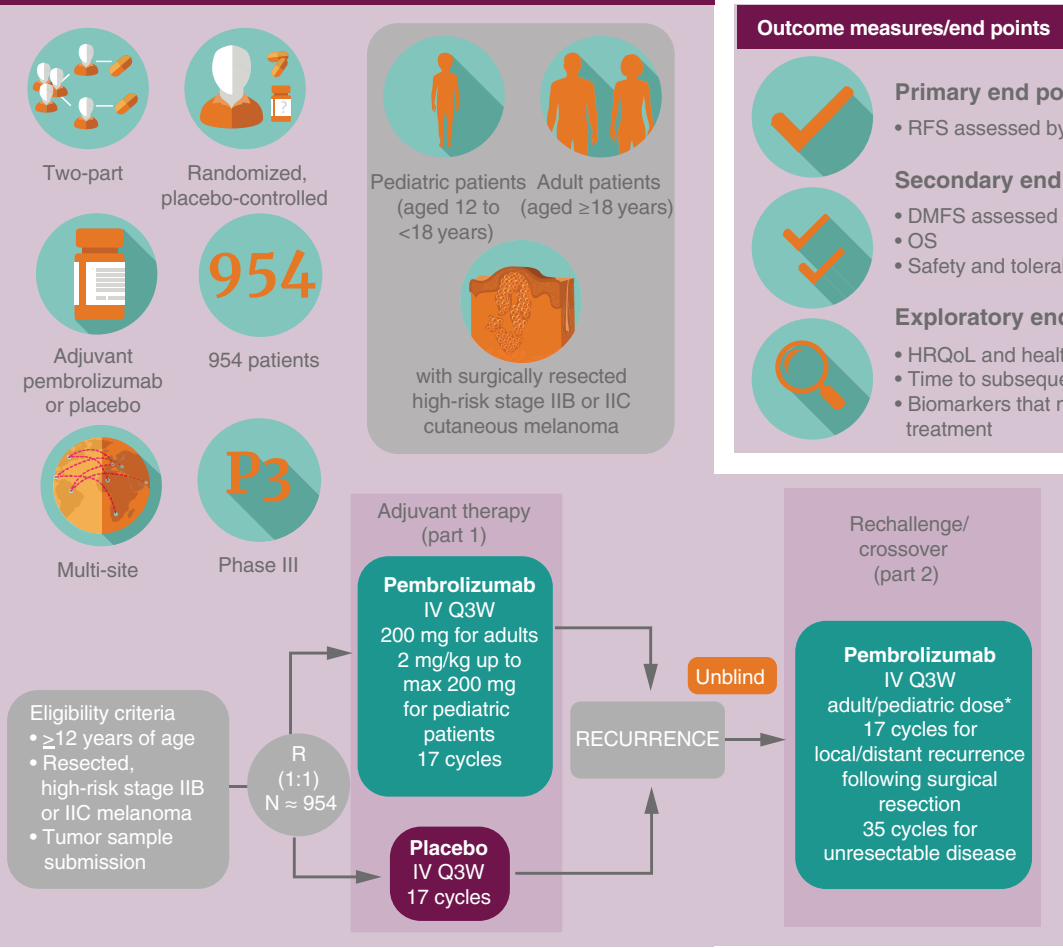

Key eligibility criteria

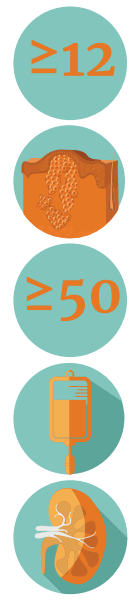

Age $\geq 12$ years healing function

Part 2 pathologist

Surgically resected and histologically/pathologically confirmed new diagnosis of stage IIB or IIC cutaneous melanoma per AJCC Cancer Staging Manual, 8th edition

$\leq 12$ weeks elapsed between complete surgical resection and first dose of study treatment and with complete wound

ECOG PS 0 or 1 in adults (patients aged $\geq 18$ years) or Lansky Play-Performance Scale score $\geq 50$ (children aged $\leq 16$ years) or Karnofsky Performance Status Scale score $\geq 50$ (children aged $>16$ years and $<18$ years)

No previous treatment for melanoma beyond complete resection of current primary melanoma lesion

Adequate hematologic, renal, hepatic and coagulation

Investigator-confirmed disease recurrence

Continuing from Part 1 placebo or and completed 17 cycles of pembrolizumab treatment with no delays in $\geq 12$ weeks

Full resection of lesions or biopsy of unresectable or metastatic disease confirmed to be melanoma by site

ECOG PS 0,1 or 2 in adults (patients aged $\geq 18$ years) or Lansky Play-Performance Scale score $\geq 50$ (children aged $\leq 16$ years), or Karnofsky Performance Status Scale score $\geq 50$ (children aged $>16$ years and $<18$ years)

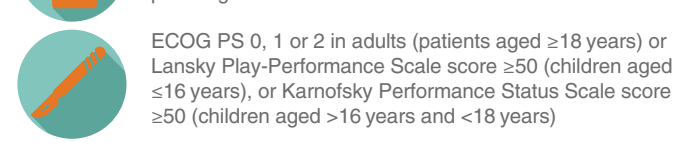

.

